The article by Kirk describes some of the shortfalls in foodborne disease surveillance in Australia, and argues for greater integration of surveillance information on the microbial contamination of food, animal carriage, human illness, and other hazards. Kirk cites the benefits that have been realised, in some Scandinavian countries, through the integration of surveillance information.

The EpiReview by Neville and McAnulty analyses the surveillance of notified enteric diseases and reports of foodborne disease outbreaks in NSW, and identifies the need to further enhance outbreak reporting.
Together, these articles provide an overview of the epidemiological and surveillance framework for the promotion of food safety in NSW. A future issue of the NSW Public Health Bulletin will explore the evolution to a single agency responsible for ensuring safe food production, the NSW Food Authority.

\section{REFERENCES}

1. Joint FOA-WHO Food Standards Programme, Codex Alimentarius Commission. Food Hygiene - Basic Texts. Rome: FOA-WHO, 1999.

\title{
FOODBORNE DISEASE SURVEILLANCE IN NSW: MOVING TOWARDS PERFORMANCE STANDARDS
}

\section{Craig Dalton}

Hunter Public Health Unit

NSW Health has sole responsibility for the surveillance of foodborne disease in humans, through the receipt of notifications for a range of conditions that are predominantly or potentially foodborne in transmission. These conditions include: salmonellosis, listeriosis, shigellosis, typhoid, Verotoxin producing E. coli infection, cholera, hepatitis A, giardiasis, and cryptosporidiosis. In addition, outbreaks of foodborne disease affecting two or more people are notifiable. Surveillance methods used in NSW are described in detail in this issue of the NSW Public Health Bulletin by Neville and McAnulty. This article describes the evolution of, and recent investments in, foodborne disease surveillance and control in NSW, and discusses the opportunities to produce measurable enhancements to food safety from these investments.

\section{THE EVOLUTION OF FOODBORNE DISEASE SURVEILLANCE IN NSW}

In 1990, the Chief Food Inspector established a position of Foodborne Outbreak Investigation Coordinator in the Food Branch of the NSW Department of Health. The Foodborne Outbreak Investigation Coordinator was given authority to investigate outbreaks of foodborne disease, utilising the resources of the Food Branch. From this time, all foodborne outbreaks were reported by food inspectors employed by the NSW Department of Health, who were functionally located throughout the state. All reports were documented centrally by the Foodborne Outbreak Investigation Coordinator. These food inspectors, and the Department's Food Branch, took the lead in the surveillance of foodborne disease.
In 1992, with the administrative transfer of food inspectors to public health units (PHUs), it became the responsibility of the PHUs to report outbreaks to the Foodborne Outbreak Investigation Coordinator in the Food Branch. An outbreak report summary form was developed to assist PHU staff to complete this requirement.

Initially, food inspectors conducted both the environmental and epidemiological investigation of outbreaks and followed up sporadic cases of salmonellosis. However, over the last 10 years, the role of food inspectors has focussed more on the environmental aspects of outbreak investigations, as epidemiologistsboth in the PHUs and the NSW Department of Health-began taking the lead on the epidemiological aspects of those investigations. This evolution continues, with the transfer, in 2004, of all NSW Health food inspectors to a new single agency responsible for ensuring safe food production in NSW, ${ }^{1}$ which will be an enhancement and an expansion of the current SafeFood Production NSW (SafeFood). However, NSW Health will retain primary responsibility for the surveillance and investigation of illness due to foodborne disease.

The infrastructure for processing data describing foodborne disease has also evolved significantly over the last 10 years. Initially, each case involved in a foodborne disease outbreak was entered into the Infectious Disease Surveillance System (IDSS) database, which later became the Notifiable Diseases Database (NDD). However, each case was entered into the database without a standard set of summary outbreak information, such as the aetiological agent, food vehicle, or setting; therefore, these data could not be analysed to identify prevention opportunities. In 2002, PHUs adopted the OzFoodNet outbreak reporting form, which captured summary outbreak information. From October 2002, PHUs 
were no longer required to enter single cases into NDD when cases were notified as members of a group affected by a foodborne outbreak. Instead, summary information on the group was captured solely with the OzFoodNet outbreak reporting form. The information is then summarised at the state level and the data is forwarded to the Commonwealth Department of Health and Aged Care for entry into the national OzFoodNet database of foodborne outbreaks. The summary information for the State has allowed the compilation and publication of useful information, as exemplified in the article by Neville and McAnulty in this issue in the Bulletin.

\section{INVESTMENTS IN SURVEILLANCE CAPACITY}

Foodborne disease surveillance is evolving in NSW, with enhancements to both the epidemiological and laboratory capacity and coordination. The appointment of a fulltime enteric disease epidemiologist in the Communicable Diseases Branch in July 2002; the appointment of a fulltime epidemiologist in OzFoodNet in September 2002; and ongoing collaboration with the Hunter Sentinel OzFoodNet site, are all signals of a significant commitment to foodborne disease surveillance and investigation in NSW. In addition, in 2003, a senior microbiologist was appointed to the position of Public Health Laboratory Liaison Officer (NSW Health), and the NSW Enteric Diseases Advisory Committee was initiated to advise NSW Health on epidemiological and laboratory issues for the control of enteric disease. The outlook for foodborne disease surveillance in NSW is excellent.

\section{Variation in outbreak reporting}

Variations in clinical practice among the area health services have become a focus of quality improvement. In the early 1970s, when marked differences in clinical practice were first identified in the United States, there was a simplistic preoccupation with identifying 'the bad apple' based on the variation documented. ${ }^{2}$ Clinical quality improvement processes have matured; variations in clinical practice are now seen as opportunities for quality improvement. ${ }^{3}$ Consequently, in 2004, variations in surveillance data should be examined, to improve public health systems.

Based on the variation in outbreak reporting rates by area health service (AHS), there are still opportunities to improve the completeness of reporting outbreaks of foodborne disease in NSW. Rates of reported foodborne outbreaks will depend on: the willingness of cases to report their illness; the history taking, testing, and notification practices of doctors; the laboratory methods employed; and the investigation and reporting practices of the PHU. ${ }^{4}$ In 2002, the number of outbreaks reported in each AHS varied from 0 to 14, a rate of 0 to 25 outbreak reports per million population. Four AHSs (24 per cent), including those with large and small populations, reported no outbreaks (Figure1). Investigation of this variation will very likely enhance the performance of our outbreak surveillance system.

\section{Timeliness of Salmonella reporting}

At the public health unit level, an analysis of the time between the collection of specimens and the receipt of

\section{FIGURE 1}

RATE OF FOODBORNE OUTBREAKS PER MILLION POPULATION AND NUMBER OF FOODBORNE OUTBREAKS REPORTED FOR 17 AREA HEALTH SERVICES, NSW, 2002

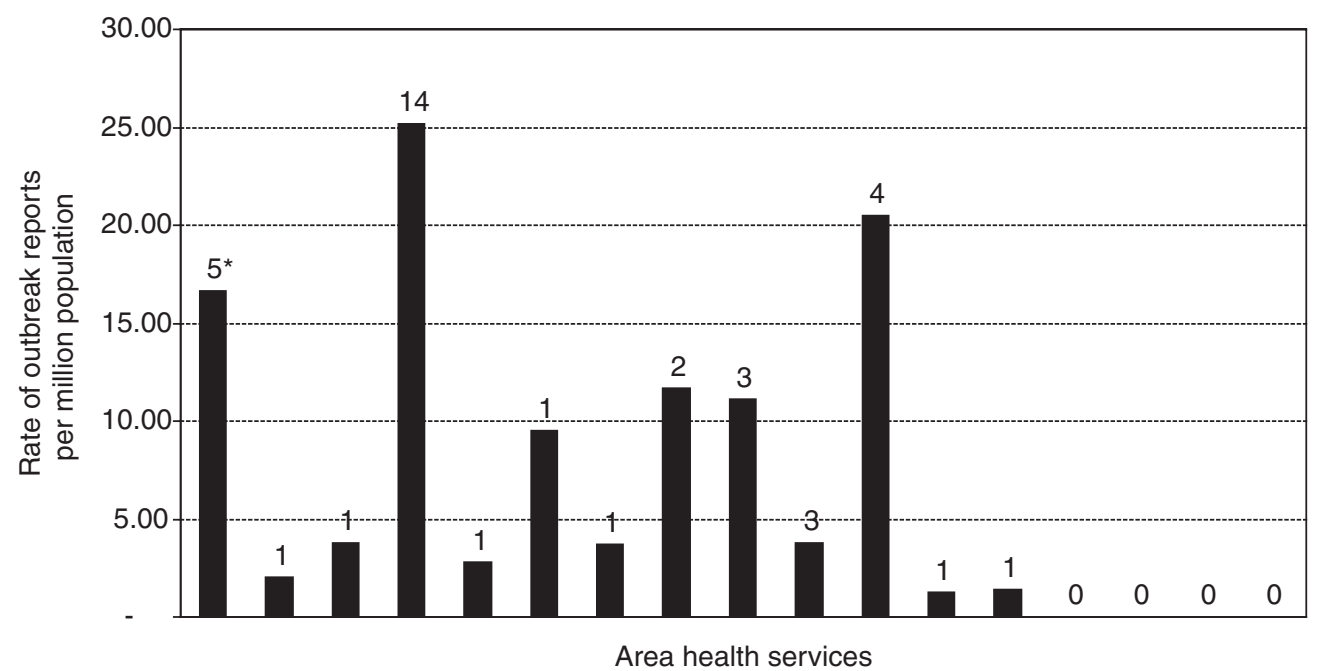

Source: Hunter OzFoodNet Sentinel Site, unpublished data. 
results of Salmonella serotype or phage type reveals significant delays - particularly in phage typing - which hamper both identification and response to outbreaks (Hunter Public Health Unit, unpublished data). Phage typing is critical for epidemiologically-meaningful clusters or outbreaks to be identified from among the more common serotypes of Salmonella, such as Salmonella Typhimurium. However, because of a range of issues including quality assurance, specialisation, economies of scale, and even convention, only two laboratories in Australia are able to phage type Salmonella and neither are in NSW. This leads to inevitable delays, as NSW laboratories package and despatch interstate approximately half of all Salmonella isolates. The Institute of Medical and Veterinary Science in Adelaide, and the Microbiological Diagnostic Unit in Melbourne, provide phage typing services, with rapid turnaround from the time the isolate arrives at their laboratories.

Questions arise as to whether another subtyping method, other than phage typing, should be applied in NSW to speed up the process; however, there are national implications that need to be considered. While a molecular subtyping method could be used in NSW to reduce the delay in recognition of outbreaks by many days, this could create the problem of splitting Australia's Salmonella subtyping systems into two that have incompatible methods. If NSW is to trial a new subtyping system, it will be important to ensure compatibility between existing phage typing and the new system, by demonstrating that it is able to map to the current system. This is required to ensure that no connection is lost with either historical surveillance data from NSW or future data from other states and overseas.

\section{The importance of Norovirus reporting}

Norovirus, previously referred to as Norwalk-like viruses, may be the most common aetiology of foodborne disease in Australia, as is hypothesised to be the case in the United States. ${ }^{5,6,7}$ While rarely fatal, the highly infectious nature of this organism can result in highly visible events. Closure of health care facilities and the cancellation of cruises are examples of the measures required to allow effective decontamination to extinguish an outbreak. Oysterassociated outbreaks of Norovirus have occurred in NSW, as have outbreaks thought to be associated with either symptomatic or convalescing foodhandlers. ${ }^{8}$ Two NSW laboratories, and other laboratories around Australia, currently offer polymerase chain reaction (PCR) testing for Norovirus, and the University of NSW has an active research program in this area. ${ }^{9}$ Because of the variability in sensitivity, and particularly sensitivity by genogroup, of this PCR test, it would be useful to have a national collaboration to identify the optimal primers, protocols, and reference reagents to better define the epidemiology of these diseases in NSW and throughout Australia. ${ }^{10}$ Newer diagnostics, such as Enzyme Immunoassay (EIA), which allow more rapid turnaround times, albeit with lower sensitivity than PCR, are becoming available and their roles need to be considered.

\section{Notification of campylobacteriosis}

As we begin to benchmark our performance against other states, it will be important to consider the issue of campylobacteriosis, as NSW is the only state in which campylobacteriosis is not notifiable. Campylobacteriosis is the most common bacterial foodborne infection in Australia, which results in significant morbidity and occasional mortality. Notification of this disease has increased dramatically in all other states and territories from 1991 to 2002 (8,813 cases to 14,619 cases, or 165 per cent $),{ }^{11}$ and it is reasonable to presume a similar increase in incidence in NSW over this time. If the national rate for 2002 of 112 cases per 100,000 is applied to the population of NSW, approximately 7,280 cases of campylobacteriosis would have occurred in NSW in 2002 compared to 2,094 cases of salmonellosis for the same period.

Many benefits could accrue to NSW if campylobacteriosis was made notifiable under the Public Health Act 1991. We could monitor trends, identify outbreaks, and study risk factors for infection through, for example, the recent national case-control study of campylobacteriosis. Additionally, national campylobacteriosis surveillance data would no longer underestimate the national burden of this disease by approximately 30 per cent.

There is concern that significant public health and laboratory resources could be consumed through notification and follow up of campylobacteriosis cases. There is also a reluctance to make these cases notifiable until direct electronic notification by laboratories to PHUs can be achieved for all notifiable conditions. However, a minimalist approach could be taken. If campylobacteriosis was notifiable, NSW Health could require quarterly or annual electronic downloads from laboratory computers to document trends in incidence and distribution by age, gender, season, and region. This would allow the monitoring of the effect of food safety programs designed to control campylobacteriosis. Promising initiatives to improve subtyping of Campylobacter isolates will enhance cluster investigations and make notification of greater public health benefit.

\section{Enhanced laboratory infrastructure}

Laboratory infrastructure is being enhanced to allow a broader range of testing for foodborne pathogens at the Institute of Clinical Pathology and Medical Research (ICPMR) in Sydney including testing for Bacillus cereus toxins, Staphylococcus aureus toxin, and Clostridium perfringens enterotoxin. In 2003, pulsed field gel electophoresis (PFGE) performed at the ICPMR has been useful in monitoring cases of listeriosis in a timely manner, to rule out any foodborne disease clusters, and PFGE will be an important public health resource in the future. The 
development of a PFGE for $C$. perfringens would help to confirm clusters, where stool collection occurs too late to allow confirmation by conventional quantification of spore counts.

\section{The role of public health units}

NSW public health units perform frontline surveillance and investigation of foodborne disease infections and outbreaks. Their location in each area health service of NSW means that they can be more reactive to local conditions. However, this demands greater coordination and cooperation to achieve statewide policy outcomes. Because this network of PHUs, with their links with local and reference laboratories, covers approximately one-third of the national population, it provides information critical to national foodborne disease surveillance and control. PHUs are moving to a performance-based quality improvement process for foodborne disease surveillance. At the August 2003 meeting of the PHU Directors Forum, a range of quality initiatives was adopted. These measures included an annual review of the timeliness of Salmonella notifications, including feedback on the delay at each laboratory, to encourage slower laboratories to improve their performance. Local protocols for triggering the initiation of outbreak investigations will be developed, and PHUs will enhance their relationships with general practitioners to promote reporting of foodborne outbreaks. To improve the timeliness and completeness of outbreak information, PHUs will fast-track the completion of OzFoodNet outbreak report forms.

\section{CONCLUSION}

The new investments in the epidemiological and laboratory infrastructure supporting foodborne disease surveillance in NSW, described in this article, should provide insights into the causes of foodborne disease in the state. The challenge will be to translate these insights into food safety policy. The launch in 2004 of a dedicated agency in NSW responsible for safe food production should provide an excellent framework for epidemiologically-driven food safety policy. A future issue of the
NSW Public Health Bulletin will focus on the role of this new agency, the NSW Food Authority.

\section{REFERENCES}

1. NSW Government. Review Required by Section 73 Food Production (Safety) Act 1998. Integration of the NSW food safety system. Sydney: SafeFood NSW, 2002. Available online at www.safefood.nsw.gov.au/issues-paper/IssuesPaper.pdf.

2. Wennberg J, Gittelsohn A. Small area variations in health care delivery. Science 1973; 182: 1102-8.

3. Kassirer JP. The use and abuse of practice profiles. $N$ Engl J Med 1994; 330: 634-636.

4. O’Brien SJ, Elson R, Gillespie IA, Adak GK, Cowden JM. Surveillance of foodborne outbreaks of infectious intestinal disease in England and Wales 1992-1999: Contributing to evidence-based food policy? Public Health 2002; 116(2): 7580.

5. Deneen VC, Hunt JM, Paule CR, et al. The impact of foodborne calicivirus disease: The Minnesota experience. J Infect Dis 2000; 181(Suppl 2): S281-3.

6. Hall JA, Goulding JS, Bean NH, et al. Epidemiologic profiling: Evaluating foodborne outbreaks for which no pathogen was isolated by routine laboratory testing: United States, 198289. Epidemiol Infect 2001; 127(3): 381-7.

7. Mead PS, Slutsker L, Dietz V, McCaig LF, Bresee JS, Shapiro C, et al. Food-related illness and death in the United States. Emerg Infect Dis 1999; 5: 607-25.

8. Stafford R, Strain D, Heymer M, et al. An outbreak of Norwalk virus gastroenteritis following consumption of oysters. Comm Dis Intell 1997; 21: 317-320.

9. White PA, Hansman GS, Li A, Dable J, Isaacs M, Ferson M, McIver CJ, Rawlinson WD. Norwalk-like virus 95/96-US strain is a major cause of gastroenteritis outbreaks in Australia. J Med Virol 2002; 68: 113-118.

10. Koopmans M, Vennema H, Heersma H, van Strien E, van Duynhoven Y, Brown D, et al. Early identification of commonsource foodborne virus outbreaks in Europe. Emerg Infect Dis 2003. Available online from www.cdc.gov/ncidod/EID/ vol9no9/02-0766.htm.

11. National Notifiable Diseases Surveillance System. Australian Department of Health and Ageing. Available online at www1.health.gov.au/cda/Source/CDA-index.cfm. Accessed on 27 November 2003. 